\title{
Optimización de portafolios accionarios a través de un micro algoritmo genético
}

Recepción: Agosto de 2007 / Aceptación: Noviembre de 2007

Mauricio Gutiérrez Urzúa Erick Torres Melillanca 3atricio Gálvez Gálvez (4) German Poo Caamaño

\section{RESUMEN}

Esta investigación muestra la optimización de portafolios accionarios mediante micro algoritmos genéticos, que resuelvan el modelo de selección de inversiones planteado por Markowitz, como una optimización multi-objetivo, en donde se maximiza la rentabilidad y se minimiza el riesgo, lo que implica, realizar una negociación entre ambos objetivos y buscar soluciones óptimas. La resolución de este problema requiere de un algoritmo genético para optimización multi-objetivo basado en óptimos de Pareto. Los resultados obtenidos señalan que esta aplicación es más eficiente que otros procesos de similares características (Non-dominated Sorting Genetic Algorithm II (NSGA II) y Pareto Archive Evolution Strategy (PAES)), pero debido al periodo de tiempo y las caracteristicas del mercado local, su poder de predicción es bajo

Palabras claves: Optimización, portafolios, Micro-algoritmo genético.

Portfolio Optimization Using A MICRO GENETICALGORITHM ABSTRACT

This research shows the portfolios optimization using micro genetic algorithms, to resolve the Markowitz's selecting investments model like a multi-objetive optimization, where is maximized profitability and minimizing the risk, thus create a negotiation between the two objectives, then find optimal solutions. To solve this problem need a genetic algorithm for multi-objetive optimization, based Pareto's optimal. The results show that this application is more efficient than other similar processes (Nondominated Sorting Genetic Algorithm II (NSGA II) and Pareto Archive Evolution Strategy (PAES)), but considering the period and the local market characteristics, its predictive power is low.

Key words: Optimization, portfolios, micro genetic algorithms.

Ind. data 10(2), 2007

\section{INTRODUCCION}

La gran mayoria de los problemas del mundo real son multi-objetivos. Estos problemas consideran varios objetivos que generalmente están en conflicto y además deben ser satisfechos al mismo tiempo. La computación evolutiva es un conjunto de algoritmos que presentan una serie de caracteristicas que permiten explorar espacios de búsqueda de soluciones óptimas, utilizando principios biológicos, cuya eficacia y eficiencia ha sido aplicada y probada en diversos problemas. Desde hace pocos años la computación natural se está aplicando en la optimización de soluciones a problemas con múltiples objetivos.

Por otra parte, se puede dividir la historia de las finanzas a partir de 1952, año en que Markowitz y otros desarrollaron estudios de selección de portafolios, diversificando las inversiones para disminuir el riesgo, estableciendo la base para inversionistas adversos al riesgo que prefieren mantener portafolios de activos, en vez de activos individuales. En este planteamiento encontramos dos objetivos en conflicto, por un lado se busca la mayor rentabilidad y por otra parte el menor riesgo, lo que genera una zona de negociación que permita encontrar el óptimo de inversión dependiendo de la tolerancia al riesgo que tengan los inversionistas. Para resolver este conflicto podemos recurrir a la programación multi-objetivos que se sustenta en algoritmos inteligentes.

Existen algunas investigaciones previas que han utilizado Algoritmos evolutivos en el área financiera. Vedarajan et al. (1997) utilizan el NSGA para Optimización de Inversión en Portafolios accionarios, en donde, dos objetivos son inicialmente considerados: maximizar el retorno esperado y minimizar el riesgo, posteriormente se agrega el objetivo de minimizar los costos de transacción. Los resultados son comparados con el uso de un Algoritmo Genético (AG) con una combinación lineal de ponderaciones y utilizando programación cuadrática. El proceso es aplicado a un portafolio de cinco grandes acciones, pertenecientes a diversas industrias, considerando un periodo de cinco años. EI NSGA y elAG con una función de agregación producen resultados similares. Ambas técnicas basadas en AG fueron consideradas mejores que la programación cuadrática por su capacidad de generar varias soluciones no-dominadas en un solo funcionamiento

Chang et. al. (1998) utilizan un AG con función de agregación para resolver problemas de optimización de portafolios, considerando como

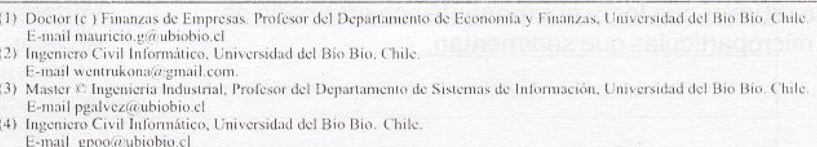


objetivos minimizar la varianza total (riesgo) y asegurar una rentabilidad para el portafolio. También considera el restringir el número de activos de inversión. Un aspecto interesante de esta aplicación es que el retorno esperado debe ser satisfecho de forma exacta. Los autores usan AG de estado constante con selección a través de torneo binario, cruza uniforme y un operador de mutación limitado.

EI AG es comparado con búsqueda Tabú y recorrido simulado utilizando la misma función de agregación. Shoaf \& Foster (1996) usan un AG con una combinación lineal de ponderaciones para selección de portafolio basado en el modelo de Markowitz, considerando dos objetivos: minimizar la varianza y maximizar el retorno. Los autores usan representación binaria, cruza mono-punto y mutación uniforme. Los resultados son comparados con el uso de un enfoque tradicional de selección de portafolios. El AG propuesto supera al método tradicional en términos de la calidad de soluciones producidas. Castro (2005) realiza un estudio comparativo de tres técnicas basadas en algoritmos evolutivos para optimización multi-objetivo y así generar múltiples soluciones (valores) de un portafolio de inversión. Para conformar las carteras toma los valores de la Bolsa de Valores de México. Las técnicas comparadas son NSGA-II, PAES y micro-AG.

Parisi et al (2004) utilizan un AG recursivo para analizar la eficiencia de los modelos multi-variados dinámicos, el cual permite predecir el signo de las variaciones semanales de los indices bursátiles IPC, TSE, DJI, y NASDAQ, entendiendo que la predicción de la dirección del movimiento del indice accionario es relevante para desarrollar estrategias de transacción efectivas. Este trabajo ha sido realizado desde un punto de vista netamente financiero, por expertos en finanzas lo que da una perspectiva diferente a la de los otros trabajos antes mencionados. El enfoque financiero da mayor validez en la aplicabilidad de los resultados obtenidos.

El objetivo de este trabajo es construir un prototipo que contenga toda la funcionalidad necesaria para resolver el problema de rentabilidad y riesgo, mediante la implementación de micro algoritmo genético. El artículo presenta el marco teórico que respalda la aplicación y la adaptación de la metodologia necesaria para resolver el problema planteado, los resultados obtenidos y las principales conclusiones.

\section{MARCOTEORICO}

Programación Multi-objetivo(PMO)

La programación multi-objetivo consiste en encontrar un vector de variables de decisión que satisfaga ciertas restricciones y optimice una función vectorial cuyos elementos representen las funciones objetivo. Estas funciones forman una descripción matemática de los criterios de desempeño que usualmente están en conflicto entre si y que se suelen medir en unidades diferentes. Por lo tanto, el término optimizar significa "encontrar una solución tal que proporcione valores para todos los objetivos que resulten aceptables para el diseñador" Osyczka (1984)

El problema de optimización multi-objetivo (POM) se define formalmente como:

Encontrar el vector $\overrightarrow{x^{*}}=\left[x_{1}^{*}, x_{2}^{*}, \ldots \ldots, x_{x}^{*}\right]^{T}$ que satisfaga las $m$ restricciones de desigualdad:

$$
\mathrm{g}_{\mathrm{i}}(\vec{x}) \leq 0 \quad i=1,2, \ldots \ldots \ldots . . . m
$$

que satisfaga las $p$ restricciones de igualdad

$$
h_{i}(\overrightarrow{x)}=0 \quad i=1,2, \ldots \ldots . p
$$

y optimice la función vectorial

$$
\vec{f}(\vec{x})=\left[f_{1}(\vec{x}), f_{2}(\vec{x}) \ldots \ldots . . . f_{k}(\vec{x})\right]^{T}
$$

donde: $\vec{x}=\left[x_{1}, x_{2}, \ldots \ldots \ldots x_{n}\right]^{T}$ es el vector de variables de decisión.

Las restricciones definen la región factible $F$ y cualquier punto en $F$ forma parte de la solución factible.

Optimizar varias funciones objetivos a la vez, no se traduce en encontrar un óptimo para cada función, sino en proponer un conjuntos de puntos en la que cada función objetivo contribuya en alcanzar una buena aptitud global, la cual será establecida y evaluada por el diseñador, un inversionista en nuestro caso. Asi el concepto de óptimo que se maneja en PMO es diferente a programar una sola función objetivo, ya que se sustenta en un compromiso entre todas las variables que satisfacen las restricciones, es decir, una solución global de la optimización. Esta noción de "óptimo" fue propuesta por Pareto en 1886 y conocida como el "óptimo de Pareto" y su definición formal es la siguiente:

Definición 1: Óptimo de Pareto

Dadas $\mathrm{k}$ funciones objetivo del problema, decimos que un punto $\vec{x} \in F$ es un óptimo de Pareto si para toda $\dot{x} \in F$, tal que para toda $\mathrm{i}=1,2 \ldots \mathrm{k}$

$$
\overrightarrow{f_{i}(\vec{x})} \leq f_{i} \overrightarrow{(x)}
$$

$y$, al menos existe una i tal que

$$
f_{i}(x)<f_{i} \overrightarrow{(x)}
$$

Esta definición dice que $\vec{x}$ es un óptimo de Pareto si no existe dentro del espacio de búsqueda $F$ un vector factible $\vec{x}$ que mejoraria algún criterio, sin hacer que 
empeore al menos otro de ellos, es decir, que lo domine, por lo que se le conoce a $\overrightarrow{x^{*}}$ como una solución no dominada.

Definición 2 Dominancia de Pareto

Un vector $\vec{u}=\left(u_{1}, \ldots \ldots . . u_{K}\right)$ se dice que domina al vector $\vec{v}=\left(v_{1}, \ldots \ldots \ldots v_{k}\right)$ denotado por $\vec{u} \prec \vec{v}$ si $\mathrm{y}$ solamente si es parcialmente menorque $v$, es decir:

$\forall i \in\{1, \ldots \ldots \ldots k\}, u_{i} \leq v_{i} \wedge \exists i \in\{1, \ldots ., k\}: u_{i}<v_{i}$

El óptimo de Pareto casi siempre produce no una, sino un conjunto de soluciones a las que se llaman no dominadas.

Definición 3: Conjunto de Óptimos de Pareto.

Dado un PMO $\vec{f}(\vec{x})$, el conjunto de óptimos de Pareto $P_{\text {true }}$ se define como:

$$
P_{\text {true }}:=\left\{\vec{x} \in F: \neg \overrightarrow{\vec{x}} \in F \vec{f}\left(x^{\prime}\right) \prec \vec{f}(x)\right\}
$$

A partir de la definición 3 se define el Frente de Pareto como el contra dominio del conjunto de puntos que forman el conjunto de óptimos de Pareto, es decir, el frente de Pareto son los valores de las funciones objetivo correspondientes a las soluciones que pertenecen al conjunto de óptimos de Pareto.

Resulta imposible determinar de manera analitica la expresión matemática que corresponde al frente de Pareto de un problema arbitrario. Aproximar dicho frente es precisamente el objetivo de la optimización multi-objetivo.

\section{Algoritmos Inteligentes}

La tendencia de las investigaciones en el área de la Inteligencia artificial han derivado en métodos de búsqueda de exploración que son un símil con modelos biológicos y fisicos que permiten resolver problemas de optimización, a continuación haremos una reseña que puede ampliarse en Fonseca y Fleming (1993), Mickelwicz (1996) Torres et al (2006).

La Computación Evolutiva (CE) es un enfoque alternativo para abordar problemas de búsqueda y aprendizaje a través de modelos computacionales de procesos evolutivos implementado a través de algoritmos evolutivos (AE). El proceso genérico de los $\mathrm{AE}$ consiste en guiar la búsqueda estocástica haciendo evolucionar a un conjunto y seleccionar de modo iterativo las mas adecuadas. La CE forma parte a su vez de un conjunto de metodologias de resolución de problemas que imitan comportamiento con mayor o menor exactitud, como las redes neuronales Hertz et al (1991), la solidificación simulada Fonseca y Fleming (1993), todas ellas se engloban bajo el término computación natural.
En general, se llama Algoritmo Evolutivo a procedimientos estocástico de búsqueda basado en el principio de evolución. Dicho principio tiene la finalidad última de la supervivencia del más apto y su forma de conseguirlo es por adaptación al entorno. Concretamente, al implementar un algoritmo a una población de individuos que representan un conjunto de candidatos a soluciones de un problema, esta es sometida a una serie de transformaciones con las que actualiza la búsqueda y después a un proceso de selección que favorece a los mejores individuos. Cada ciclo de transformación más la selección, constituye una generación.

Para simular el proceso de evolución, un $\mathrm{AE}$ requiere de:

1. Una población de posibles soluciones debidamente representadas a través de individuos.

2. Un procedimiento de selección basado en la aptitud de los individuos.

3. Un procedimiento de transformación, esto es, de construcción de nuevas soluciones a partir de las disponibles actualmente.

Los algoritmos genéticos (AG) que se han desarrollado bajo el esquema evolutivo, hacen evolucionar una población inicial que esta codificada como enteros binarios, sometiéndola a transformaciones unitarias y binarias genéricas. dentro de un proceso de selección. Asi dentro de la implantación de un algoritmo genético es crucial definir el proceso de selección, el proceso de reproducción que hacen uso de los operadores genéticos.

Tratamiento de problemas Multi-objetivo con técnicas evolutivas

El área de investigación nombrada optimización evolutiva multi-objetivo, se basa en la búsqueda de soluciones utilizando un algoritmo evolutivo (AE) como una forma alternativa para resolver problemas que presentan varios objetivos a la vez. La primera implementación de un algoritmo evolutivo multiobjetivo fue presentado por Shaffer (1985), Vector

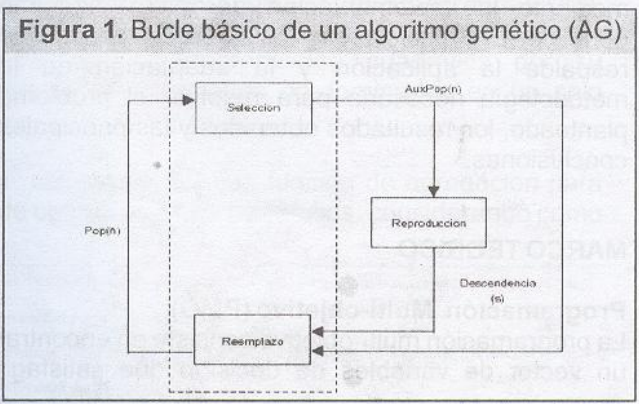


Evaluated Genetic Algorithm (VEGA), que corresponde a la primera generación de algoritmos de este tipo entre cuales se cuenta también NSGA Deb et al (2002), NPGA Horn et al (1994) y MOGA Fonseca y Fleming (1993).

La implementación del mecanismo de elitismo en el contexto de optimización multi-objetivo, se realiza a través de una población secundaria, en la que se almacenan los individuos no dominados, encontrados durante el proceso de búsqueda. Dentro de los algoritmos de segunda generación nos interesan por su buen comportamiento y aplicaciones en el área de las Finanzas, los desarrollados por Castro (2005) Srinivas y Deb (1994) y Coello y Toscano (2001).

Modelo de Markowitz como Problema de Programación Multi-objetivo

El modelo de Media- Varianza de Markowitz (1952) se define como la optimización simultánea de dos funciones objetivo.

Rendimiento del portafolio

$\operatorname{Max} E\left(R_{p}\right)=\sum^{\prime \prime} w_{i} \times E\left(R_{i}\right)$

Riesgo del Portafolio

$$
\begin{aligned}
& \text { Mino }_{i=}^{2}=\sum_{i=1}^{n} \sum_{i=1}^{n} w_{i} \times w_{i} \times p_{i j} \times p_{j} \times p_{i} \\
& \text { s.a } \\
& \sum_{i=1}^{n} w_{i}=1 \\
& w_{i} \geq 0
\end{aligned}
$$

Después de una revisión de las referencias bibliográficas de la aplicación del enfoque del paradigma evolutivo se plantean las siguientes consideraciones:

La primera pregunta que surge, es por que no diseñar tan solo un algoritmo que solucione el problema cuadrático propuesto inicialmente por Markowitz. Al respecto Vedejaran et al (1997) señala que para un

Figura 2: Descomposición generalizada de las tareas de los AE. (AG)

Tareas Generales de los AE

1. Inicializar Poblacion

2 Evaluar Aolizdes

4. Mutacion

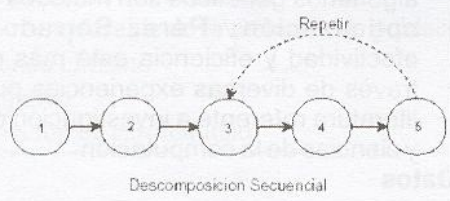

Mauricio Gutiérrez U., Erick Torres M., Patricio Gälvez G. y Germán Poo C. 》〉

portafolio de tamaño $\mathrm{n}$, el conjunto de datos de entradas es $\left(2^{*} n\right)+\left(n^{*}(n-1) / 2\right)$, por lo que si $n$ es grande puede ser laborioso seguir la pista de todos los coeficientes de correlación, y se pueden generar problemas por restricciones de almacenamiento de memoria. Además y mas restrictivo que lo anterior, es que para resolver el problema, es necesario que la matriz de covarianzas sea positiva y si existen discrepancias numéricas (por ejemplo imprecisión por redondeo), esta suposición podria ser violada y no se podria resolver. Esto no es improbable con datos reales particularmente cuando $n$ es muy grande, dado que las covarianzas son estimaciones de series de tiempo de los precios de los instrumentos financieros reales.

Por otro lado, debido al escaso uso del modelo, éste no considera aspectos como el costo de transacción por cambios en el portafolio y una mayor diversificación del portafolio.

Para incorporar la diversificación del portafolio es necesario agregar la restricción.

$\forall i \in\{1,2, \ldots n\}: w_{i} \leq W_{\max }$ donde $w_{\max }$ es una constante.

La variante que considera el costo de transacción por cambios en el portafolio (rebalanceo), causa problemas al algoritmo cuadrático, cuestión que puede ser resuelta en el enfoque multi-objetivo introduciendo una tercera función objetivo a ser minimizada

$$
f_{\cos t}=\sum_{i=1}^{n} c_{i} \times\left(w_{i}-w_{i}^{*}\right)^{?}
$$

donde: $w_{i}^{*} \in R_{+}^{0}$ es la participación inicial del instrumento financiero $i$ en el portafolio que va a ser potencialmente cambiado, debido a transacciones por rebalanceo y la constante $c ; R$ es el costo de transacción del instrumento financiero i

Micro Algoritmo Genético para Optimización Multi-objetivo

El Micro Algoritmo Genético para Optimización Multiobjetivo (micro-AG), fue creado y desarrollado por Toscano (2001), técnica utilizada en este estudio por las siguientes razones:

1. Es altamente competitiva

2. Es eficiente.

3. Se basa en novedosas tendencias en optimización multi-objetivo

4. No presenta mayores dificultades de implementación.

El Micro-AG es un algoritmo genético con una población de tamaño muy pequeño y un proceso de re-inicialización, combinado con un archivo externo para guardar los vectores no-dominados encontrados previamente y un mecanismo para mantener 
diversidad, basado en distribución geográfica, para resolver problemas de optimización multi-objetivo de diferentes grados de complejidad.

Esquema general del micro-AG.

Inicialmente se llena la memoria de población $(P)$ con individuos generados aleatoriamente. Esta memoria está dividida en dos partes: una reemplazable $(P r)$ y otra no reemplazable $(P n r)$. De esta memoria, el micro-AG obtendrá una pequeña población de trabajo $(P t)$ para evolucionarla. Tras la ejecución del micro-AG se realiza un filtro, para eliminar las soluciones dominadas y finalmente quedarse con sólo dos soluciones (S1) y (S2). Posteriormente éstas se comparan contra un par de contendientes, $\mathrm{si}$ existe más de una solución no-dominada de (Pr). Si (S1) o (S2) dominan a sus adversarios; ocupan un lugar en $(P r)$ y finalmente tanto $(S 1)$ como $(S 2)$ se insertan en el archivo histórico. Comienza el ciclo otra vez, hasta que se alcanza la condición de término. La evolución de $(P t)$ por parte del micro-AG es realizada en forma tradicional: Se realiza selección, cruza, mutación y elitismo hasta que se alcanza la convergencia nominal. En el caso de micro-AG la convergencia nominal se alcanza cuando se ha cumplido cierto número de iteraciones.

\section{METODOLOGÍA}

El problema a resolver

La Teoría de Selección de Portafolios de Markowitz (1952) tiene caracteristicas propias, tanto en su definición como en su solución, ya que, sus

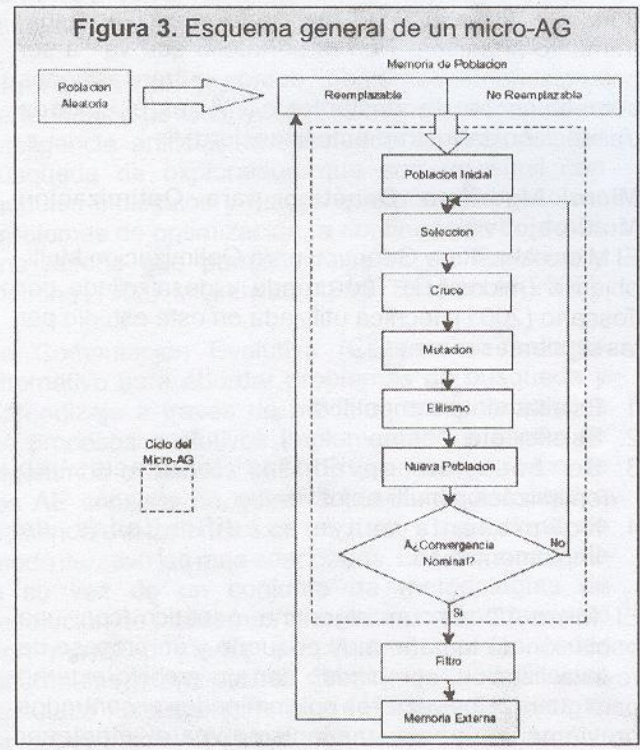

supuestos permiten el modelado matemático, a partir de los cuales se establecen los requerimientos para obtener buenas soluciones Castro (2005)

Características del problema

La elección de la frontera eficiente, es un problema de optimización multi-objetivo (POM) que se caracteriza por la variedad de activos financieros, y los porcentajes de riqueza invertida en cada uno de ellos, lo cual hace que el espacio de búsqueda formado por sus posibles combinaciones crezca de manera exponencial.

El problema planteado por Markowitz plantea los siguientes requerimientos:

1. Dado un conjunto de activos financieros, mostrar una perspectiva de las mejores combinaciones posibles de rentabilidad y riesgo.

2. Dado un nivel de riesgo, obtener la máxima rentabilidad.

3. Dado un nivel de rentabilidad, obtener el minimo riesgo.

Propuesta de solución

Se propone la construcción de una aplicación que por medio de un Algoritmo Evolutivo para Optimización Multi-objetivo permita la resolución del problema de Media-Varianza. La técnica a utilizar es el Micro Algoritmo Genético para Optimización Multi-objetivo.

\section{Fundamentos de la solución}

1. El problema de Markowitz puede ser planteado de una forma multi-objetivo.

2. Existen grandes similitudes en conceptos fundamentales entre la teoria de portafolio y la optimización multi-objetivo. En la teoria de portafolio se introdujo el concepto de dominación que establece que es preferible la cartera con menor riesgo; y de la misma manera, para cada clase de riesgo es preferible la inversión con mayor rentabilidad, tipo Pareto (1896). El Frente de Pareto, que representa el conjunto de los mejores valores para las funciones objetivo que se pretenden optimizar en distintas combinaciones de cada una de ellas, lo cual corresponde al concepto de Frontera Eficiente que es el conjunto de valores óptimos para el problema de MediaVarianza, ya que está compuesto por los portafolio con las rentabilidades más altas para cada nivel de riesgo.

3. La computación evolutiva, y en particular los algoritmos genéticos son métodos de búsqueda y optimización, Pérez-Serrado (1996), su efectividad y eficiencia está más que probada a través de diversas experiencias publicadas en la literatura referente a investigación de operaciones y ciencias de la computación. 
Es necesario contar con un volumen de datos que abarque un periodo importante de años y se requiere que estos datos se presenten de manera uniforme. Para realizar esta investigación se trabajó con las acciones que se transan en la bolsa de Valores de Santiago de Chile y se definió un periodo de tiempo de 10 años (1996 2005), 7 años para evaluar el comportamiento de los portafolios y 3 años para evaluar el valor predictivo del modelo. Se consideró una periodicidad de dos meses, quedando setenta y cuatro activos para realizar el ejercicio. Se realizaron dos procesos, uno con una cartera sin restricciones de cardinalidad y otra con una cartera compuesta por 12 activos Evans y Archer (1968).

\begin{tabular}{|l|l|l|l|}
\hline 01. BANMEDICA & 02. CALICHERAA & 03. CAMPOS & 04. CAP \\
05. CAROLINA-A & 06. CCT & 07. CEMENTOS & 08. CERVEZAS \\
09. CGE & 10. CHILECTRA & 11. CHOLGUAN & 12. CIC \\
13. CLUBUNION & 14. CMPC & 15. COLBUN & 16. COLOSO \\
17. CONCHATORO & 18. COPEC & 19. CREDITO & 20. CRISTALES \\
21. CTC - A & 22. CTC - B & 23. CTI & 24. CUPRUM \\
25. EDELMAG & 26. EDELNOR & 27. ELECDA & 28. ELECMETAL \\
29. ELILSA & 30. EMELARI & 31. EMELSA & 32. EMLIANA \\
33. END ESA & 34. ENERSIS & 35. ENTEL & 36. EPERVA \\
37. FOSFOROS & 38. GASCO & 39. GENER & 40. HABITAT \\
41. HIPICO & 42. IANSA & 43. INDIVER & 44. INFORSA \\
45. INMURBANA & 46. LAN & 47. MADECO & 48. MARINSA \\
49. MASISA & 50. MELON & 51. MINERA & 52. PACIFICO \\
53. MASUR & 54. PEHUENCHE & 55. PILMAIQUEN & 56. PIZARRENO \\
57. POLO & 58. PORTADA-B & 59. PROVIDA & 60. PUCOBRRE-A \\
61. RIO MAIPO & 62. SAN PEDRO & 63. SANTARITA & 64. SANTAMARIA \\
65. SIEMEL & 66. SINTEX & 67. SIPSA & 68. SM -CHILEA \\
69. SM-CHILEB B & 70. SOMELA & 71. TE LSUR & 72. VAPORES \\
73. VENTANAS & 74. ZOFRI & & \\
\hline
\end{tabular}

\section{Etapas del proceso}

1. Definición de funciones objetivos

$$
\begin{gathered}
\text { maximizar } \boldsymbol{E}\left(\boldsymbol{R}_{p}\right)=\sum_{i=1}^{n} w_{t} \cdot \boldsymbol{E}\left(\boldsymbol{R}_{i}\right) \\
\text { minimizar } \sigma_{p}=\sqrt{\sum_{i=1}^{n} \sum_{f=1}^{n} w_{i} \cdot w_{f} \cdot \sigma_{i f}}
\end{gathered}
$$

sujeto a:

$$
\begin{gathered}
\sum_{i=1}^{n} w_{i}=1 \\
n \leq 12
\end{gathered}
$$

$\mathrm{n}$ = número de activos a invertir (restricción de cardinalidad)

\section{Diseño del cromosoma}

Los cromosomas que se utilizan en este trabajo están compuestos por 74 genes, uno para cada activo financiero. Cada gen representa el porcentaje de riqueza invertido para cada activo del portafolio. Existen varias posibilidades de tamaño para los genes, lo cual establece la cantidad de valores dentro del rango especifico, en este caso entre cero y uno, que puede representar cada gen y por lo tanto la precisión de cada instrumento financiero y el modelo.

\section{Cuadro 1. Parámetros para la ejecución del micro-AG}

\section{Representación}

Número de genes

Tamaño del gen

Tamaño del cromosoma

Generaciones

Tipo de selección

Probabilidad de cruza

Tipo de cruza

Probabilidad de mutación

Probabilidad de mutación Knapsack

Tipo de mutación

Divisiones del espacio de Solucion es

Tamaño del Frente de Pareto

Tamaño de la Memoria de Población

Porcentaje no reemplazable de la memoria de población

Ciclo del segundo elitismo

Funciones Objetivo

Valor de restricción de cardinalidad

Binaria

74

20 bits

1480 bits

1000
Torneo binario con base en dominancia y relación rentabilidad / riesgo

$50 \%$

2 puntos

$3 /($ bitsdel cromosoma $)=3 / 1480=0,002027027$

$3 /$ genesdel cromosoma $)=3 / 74=0,040540541$

Bit a bit. Para la mutación Knapsack gen a gen 15

140 individuos

70 individuos

$30 \%$

24

2

12 


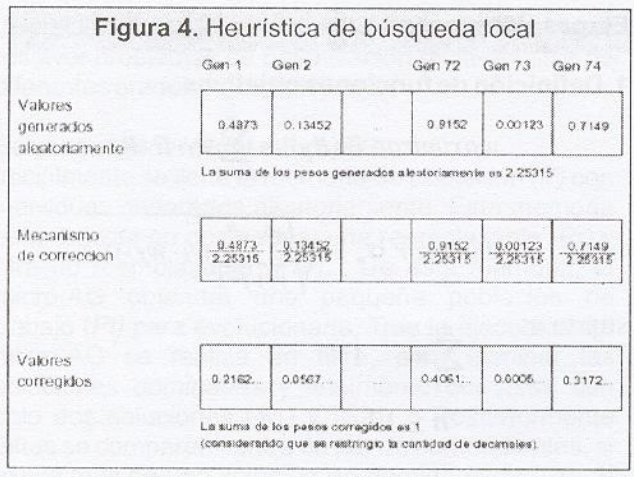

3. Problema de mochila o similitudes a Knapsack Teniéndose $n$ objetos distintos y una mochila, cada uno de los objetos tiene asociado un peso positivo wiy un valor positivo vi para $i=1,2, \ldots . n$. La mochila puede llevar un peso que no sobrepase $W$. Teniendo en cuenta estos datos, el objetivo es llenar la mochila de tal forma que se maximice el valor de los objetos transportados en ella. Hay que considerar que la suma de los pesos no debe superar la capacidad máxima $W$ de la mochila.

$$
\text { Maximizar } \sum_{1}^{n} x_{1}
$$

sujeto a:

$$
\sum x_{i} w \quad w
$$

donde

$$
\begin{gathered}
r>0 \\
w_{i}>0 \\
x, 10.1 ! \\
i \quad 1 \quad n
\end{gathered}
$$

\section{Heurística de búsqueda local}

El funcionamiento de la Heuristica de búsqueda local está representado por la Figura 4.

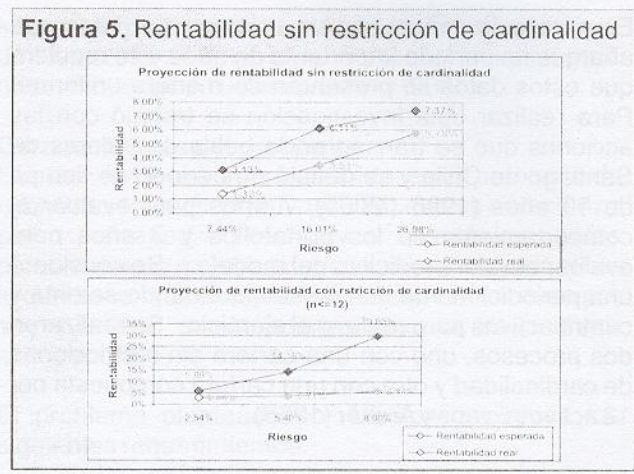

\section{Parámetros del algoritmo}

Los parámetros se muestran en el Cuadro 1.

\section{RESULTADOS}

Los Cuadros 2, 3 y 4 muestran los resultados del estudio desarrollado.

\section{CONCLUSIONES}

El desempeño de esta aplicación experimentó un notable progreso en la etapa de ensayo, en la cual el algoritmo fue probado en distintas situaciones, las cuales generaron algunas modificaciones del modelo presentado como diseño inicial.

De las mejoras realizadas, gran parte fueron producto de la observación empírica, motivada por la significancia de los cambios producidos, y también por la necesidad de reducción del tiempo de ejecución del algoritmo.

El resultado arrojó un rendimiento superior del micro-

\begin{tabular}{|c|c|c|c|}
\hline \multirow[t]{2}{*}{$\begin{array}{l}\text { Cantidad de generaciones } \\
150\end{array}$} & \multirow[t]{2}{*}{$\begin{array}{l}\text { Tiempo ejecución } \\
5 \text { horas } 14^{\prime} 27^{\prime \prime}\end{array}$} & \multicolumn{2}{|c|}{$\begin{array}{cc}\text { Tamaño Frente Pareto } & \text { Valores extremo } \\
\end{array}$} \\
\hline & & & Rentabilidad: $3.55 \%$ Riesgo: $8.69 \%$ \\
\hline \multirow[t]{2}{*}{500} & 7 horas 1 " 44 " & 8 & Rentabilidad: $3,35 \%$ - Riesgo: $7,0 \%$ \\
\hline & & & Rentabilidad: $5,34 \%$ Riesgo: $14,94 \%$ \\
\hline \multirow[t]{2}{*}{1000} & 16 horas $47^{\prime} 18^{\prime \prime}$ & Rentabili & dad: $3,13 \%-$ Riesgo: $6,74 \%$ \\
\hline & & & Rentabilidad: $4,12 \%$ Riesgo: $10,07 \%$ \\
\hline
\end{tabular}
AG comparándolo con otros algoritmos, ya que en varias de las pruebas realizadas presentó un mejor rendimiento que otros más estudiados en el tiempo como el Non-dominated Sorting Genetic Algorithm II (NSGA II) y Pareto Archive Evolution Strategy 
Tabla 3. Tamaño frente de Pareto sin restricción de cardinalidad

\begin{tabular}{|c|c|c|}
\hline Cantidad de generaciones & Tiempo de ejecución & Tamaño frente de Pareto \\
\hline 50.000 & 43 minutos & 66 \\
\hline 100.000 & 1 hora 30minutos & 57 \\
\hline 200.000 & 2 horas 9 minutos & 77 \\
\hline
\end{tabular}

(PAES). Cuando se realizan pruebas con funciones objetivo que tienen restricciones, el micro algoritmo genético presente su rendimiento más bajo.

Lamentablemente, en el mercado chileno, la información histórica es escasa para los requerimientos del modelo. El hecho de trabajar con una restricción de siete años, hace que el modelo no pueda predecir con suficiente precision e comportamiento de los portafolios de inversión, ver Figuras 5 y 6 . Analizando las proyecciones de rentabilidad se observó un proceso de sobreestimación de las rentabilidades en los distintos niveles de riesgo, especialmente en la construcción de portafolios con restricciones de cardinalidad.

Es necesario considerar como aspecto que atenta contra la fiabilidad del modelo la periodicidad de las observaciones y las caracteristicas particulares del periodo que se utilizó para la estimación de las rentabilidades. La aplicación construida no es un tomador automático de decisiones financieras que permite elegir en forma mecánica que portafolio de inversiones es el más conveniente para invertir, sino que un prototipo que contiene toda la funcionalidad necesaria para resolver el problema de rentabilidad y riesgo. El Administrador financiero deberá evaluar que ponderación le dará a los resultados de este estudio con respecto a las otras variables que maneja para tomar sus decisiones.

Esta investigación abre una interesante puerta hacia futuros proyectos que permitan simular y optimizar decisiones financieras, desarrollando nuevos modelos y adaptándolos a las caracteristicas y limitaciones de cada mercado en particular.

\section{REFERENCIAS BIBLIOGRAFICAS}

1. Castro, J. (2005). Creación de Portafolios de Inversión Utilizando Algoritmos Evolutivos Muti- objetivo. Tesis de Maestria en Ciencias, Centro de Investigación y Estudios Avanzados del Instituto Politécnico Nacional de México.

2. Chang, T.; Jeade, N. y Bealey, J. (1998). Heuritcs for Cardinality Constrained Potfolio Optimization. Tecnical report, The Management School, Imperial College, London SW72A2 England.

3. Coello, C. y Toscano, G. (2001). A Micro Genetic Algorihm for Multiobjetive Optimizacion. First Internacional Conference on Evolutionary MultiCriterion Optimization. Springer Verlag. Lecture notes in Computer Science.

4. Deb, K.; Amrit, P.; Agrawal, S.; Pratab, A. y Meyarivan, T. (2002). A Fast and Elitist Multiobjetive Genetic Algorithm: NSGA II. IEEE Transactions on Evolutionary Computation, 6 (2): 182-197.

5. Evans, J. y Archer, S. (1968). Diversification and the Reduction of Dispersion: An Empirical Analysis. The Journal of Finance, $23(5)$ : 761 767.

6. Fonseca, C. y Fleming, P. (1993). Genetic Algorithms for Multiobjetive Optimization. Fifth International Conference on Genetic Algorithms. University of Illinois USA.

7. Hertz, J.; Krogh, R. y Palmer, R. (1991). Introduction to the Theory of Neural Computations. $1^{\text {a }}$ ed. Addison-Wesley Publishing Company. Boston. USA,

8. Horn, J; Nafpliotis, N. y Goldberg, D. (1994). A Niched Pareto Genetic Algorithm for Multiobjetive Optimization. Proceedings of the First IEEE Conference on Evolutionary Computation, IEEE World Congress on Computational Intelligence. 1:82-87.

Tabla 4. Tamaño frente de Pareto con restricción de cardinalidad de 12 activos por portafolio

\begin{tabular}{|c|c|c|}
\hline Cantidad de generaciones & Tiempo de ejecución & Tamaño frente de Pareto \\
\hline 50.000 & 1 hora 12 minutos & 58 \\
\hline 100.000 & 2 horas 29 minutos & 80 \\
\hline 200.000 & 2 horas 54 minutos & 75 \\
\hline
\end{tabular}


9. Markowitz, H. (1952). Portfolio Selection. Journal of Finance 7 (1): 77-91.

10. Michelwicz, Z. (1996). Genetic algorithms+ Data Structures $=$ Evolutionary programs. $3^{\mathrm{a}}$ ed Springer.

11. Osyczka, A. (1984). Multicriterion optimization in engineering with Fortran Programs. $1^{\mathrm{a}}$ ed. Ellis Horwood Limited.

12. Pareto, W. (1896). Course de Economie Politique, Volume I \& II. F. Rouge, Lausanne. Francia.

13. Parisi, F.; Parisi, A. y Cornejo, E. (2004) Algoritmos Genéticos y Modelos Multivariados Recursivos en la Predicción de Índices Bursátiles de América del Norte: IPC, TSE, NASDAQY DJI. El Trimestre Económico N ${ }^{\circ} 284$ : 789-810.

14. Pérez-Serrada, A. (1996). Una Introducción a la Computación Evolutiva. Tesis de Maestria. Universidad de Valladolid. España.

15. Schaffer, J. (1985). Multiobjetive Optimization with Vector Evaluated Genetic Algorithms. First
Internacional Conference on Genetic Algorithms 93-100.

16. Shoaf J S; Foster JA (1996)A Genetic Algorithms Solution to the Efficient Set Problem: A Tecnique for Portfolio Selection Based on the Markowitz Model. In Proceeding of the Decision Sciencies Institute, Annual Meeting Orlando Florida, USA.

17. Srinivas, N; Deb, K. (1994). Multiobjetive Optimization Using Nondominated Sorting in Genetic Algorithms. Evolutionary Computation 2(3): 221-248

18. Torres, E. Gutiérrez, M. Gálvez, P. (2006) Algoritmo Genético (Micro-AG) en Optimización de Carteras. VI Conferencia Internacional de Finanzas, Universidad de Santiago de Chile.

19. Toscazo, G. (2001). Optimización Multi-objetivo Usando un Micro Algoritmo Genético. Tesis de Maestría. Universidad Veracruzana LANIA, México.

20. Vederajan, G.; Chi Chan, L. y Goldberg D. (1997). Investment portfolio optimization using genetic algorithms. Genetic Programing Conference Sandford University. USA. 